\title{
Sensory evaluation of bitterness and astringency sub-qualities of wine phenolic compounds: synergistic effect and modulation by aromas
}

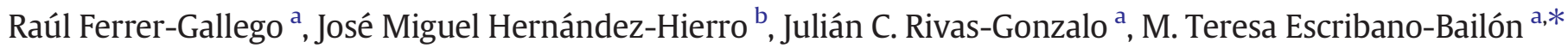 \\ a Grupo de Investigación en Polifenoles, Unidad de Nutrición y Bromatología, Facultad de Farmacia, Universidad de Salamanca, Campus Miguel de Unamuno, E 37007 Salamanca, Spain \\ ${ }^{\mathrm{b}}$ Food Colour E' Quality Laboratory, Department of Nutrition E' Food Science, Universidad de Sevilla, Facultad de Farmacia, 41012 Sevilla, Spain
}

\section{A R T I C L E I N F O}

Article history:

Received 7 February 2014

Accepted 29 May 2014

Available online 6 June 2014

\section{Keywords:}

Phenolic compounds

Sensory analysis

Bitterness

Astringency

Aromas

Wine

\begin{abstract}
A B S T R A C T
Mouthfeel characteristics of wine phenolic compounds (catechin, epicatechin, coumaric acid, caffeic acid, protocatechuic acid and gallic acid) were evaluated by sensory analysis using a Labeled Magnitude Scale for rating the intensity of the perceived sensations. Synergisms on bitterness and on astringent sub-qualities were detected when the phenolic compounds were tested as mixtures in comparison to individual compounds, maintaining the total amount of stimulus constant in all tasted samples. Principal component analysis was applied to observe trends in the data and to indicate relationships between phenolic substances and sensory attributes. It allowed us to easily visualize the synergistic effect discussed above. To our knowledge, this is the first time that astringency synergism between phenolic compounds has been described. This research also shows that wine aromas may modulate the perception of the astringency. In this study, the addition of volatile compounds increased the intensity of the astringent perception of the flavanol solutions and also its persistence.
\end{abstract}

(c) 2014 Elsevier Ltd. All rights reserved.

\section{Introduction}

Phenolic compounds are plant metabolites widely spread in food and beverages such as wine and tea. They contribute to important organoleptic properties (color, bitterness and astringency) although many gaps still exist concerning the compounds and the mechanisms that determine the different mouthfeel characteristics. One important factor contributing to these gaps is the large amount of compounds implicated in the sensory perception. The astringent perception can be produced by different kinds of compounds including metal salts, phenolic compounds, acids and dehydrating agents such as alcohols. Just among phenolic compounds, there are more than 8000 structures, from the simplest to the most complex ones. The simplest structures are phenolic acids (hydroxybenzoic and hydroxycinnamic acid derivatives), consisting of a single benzene ring. Little research has been reported on the individual sensory profile of these compounds although it is well documented that they contribute to the bitter and astringent oral sensation of food and beverages (Challacombe, Abdel-Aal, Seetharaman, \& Duizer, 2012; Hufnagel \& Hofmann, 2008).

In addition to the broad type of phenolic compounds that could contribute to the mouthfeel, it should be considered that the mechanisms of astringency are not well understood yet. Many studies have been focused on the interactions between phenolic compounds and proteins as

\footnotetext{
* Corresponding author at: Grupo de Investigación en Polifenoles, University of Salamanca, Spain. Tel.: + 34923294537.

E-mail address: escriban@usal.es (M.T. Escribano-Bailón).
}

mechanisms that could explain the perception of the astringency (de Freitas \& Mateus, 2012). Recently, a specific interaction between tannins and salivary proteins that leads or not to the precipitation of the complex depending on the colloidal state of the tannin has been demonstrated (Cala et al., 2012). It has also been suggested that the precipitation of salivary proteins does not explain all the quality aspects of the astringency (Ferrer-Gallego, Gonçalves, Rivas-Gonzalo, Escribano-Bailon, \& de Freitas, 2012; Schwarz \& Hofmann, 2008) and that multiple mechanisms can take place simultaneously (Gibbins \& Carpenter, 2013).

Predictive models using different techniques such as fluorescence, capillary electrophoresis, enzymatic assays, microcalorimetry and nephelometry, among others have also been developed as an alternative approach to traditional sensory analysis (de Freitas \& Mateus, 2012). However, sensory analysis remains being a useful tool for evaluating sensory properties and it is a unique form in describing the mouthfeel qualitative characteristics at the present time (Ferrer-Gallego, GarcíaMarino, Hernández-Hierro, Rivas-Gonzalo, \& Escribano-Bailón, 2010; Ferrer-Gallego, Hernandez-Hierro, Rivas-Gonzalo, \& Escribano-Bailon, 2011; Gawel, Oberholster, \& Francis, 2000). It should also be noted that the large terminology for communicating the high number of the astringent sub-qualities (Gawel et al., 2000) makes the assessment of the attributes by analytical methods very difficult.

Additionally, the oral feel could be different depending on several factors such as $\mathrm{pH}$, acidity, percentage of ethanol, content of carbohydrates, and saliva characteristics (de Freitas \& Mateus, 2012) and also on the physiological variations of parotid gland functionality (Dinnella, Recchia, Vincenzi, Tuorila, \& Monteleone, 2010). All of the above contributes to the controversy regarding the structure of the 
phenolic compounds responsible for the elicited mouthfeel in certain matrices. In wines, Hufnagel and Hofmann (2008) have reported that the fractions that mainly contributed to puckering and velvety astringency included hydroxybenzoic and hydroxycinnamic acids. The monomeric flavan-3-ols, procyanidin dimers and trimers do not seem to be of major importance of the astringent perception (Hufnagel \& Hofmann, 2008), which is contrary to other studies (Kallithraka, Bakker, \& Clifford, 1997; Peleg, Gacon, Schlich, \& Noble, 1999; Robichaud \& Noble, 1990; Thorngate \& Noble, 1995).

In addition, it should be considered that interactions between wine compounds can affect its aroma, flavor and mouthfeel (Jones, Gawel, Francis, \& Waters, 2008). That is why the possible relationship between aromas and astringency should be evaluated. Aroma substance retention by catechin has been studied (Dufour \& Bayonove, 1999) and it has been observed that variations in total concentration of polyphenols could lead to significant loss of aroma compounds through intermolecular interactions. The supramolecular complexation is dependent on the physicochemical structure of the phenolic compound. The mechanism involved in the interaction is an intricate combination of weak noncovalent interactions, of which hydrogen bonding makes a major contribution to the specific conformational geometry (Jung, de Ropp, \& Ebeler, 2000). It has been stated that the intensities of fruity and floral aromas seem to decrease when the level of polyphenols increases (Goldner, Lira, van Baren, \& Bandoni, 2011) and that the addition of grape seed extracts to wine not only changes astringency, but also enhances the woody/earthy aroma and reduces the fruity aroma (Cliff, Stanich, Edwards, \& Saucier, 2012)

The influence of the volatile compounds on the perception of the astringency has not been widely reported. It has been stated that the addition of volatile fruity extracts reduces the astringency and the bitterness of wines (Saenz-Navajas, Campo, Fernandez-Zurbano, Valentin, \& Ferreira, 2010). In yogurt, it has been demonstrated that the olfactory perception enhances the astringency and influences their mouthfeel (Kora, Latrille, Souchon, \& Martin, 2003). In the same way, it has been recently demonstrated that the apple texture perception can be modified by the apple odorant extract (Charles et al., 2013). The perception of the astringency could be modulated by volatile compounds due to a cognitive association between both, since 'taste' involves olfaction, gustation and chemesthesis. Thus, some aromas could enhance the saltiness taste, allowing the reduction of sodium chloride content in food (Lawrence, Salles, Septier, Busch, \& Thomas-Danguin, 2009).

From our point of view, the evaluation of the astringent sub-qualities of phenolic compounds in simple models is the beginning to be able to know if these compounds can contribute on the astringent perception of food and beverages. Other studies have studied the binary taste mixture interactions that explain synergisms in sweetness, bitterness, sourness and umami taste (Morita, Narukawa, \& Hayashi, 2007; Prescott, Ripandelli, \& Wakeling, 2001) but to the best our knowledge no research has been focused on the phenolic mixtures and astringent sub-qualities.

The main purpose of this work was to evaluate and compare the bitterness and the astringent sub-qualities of catechins and phenolic acids by sensory analysis, individually and as mixtures. Moreover, the influence of the volatile compounds on the perception of the astringency was also evaluated.

\section{Material and methods}

\subsection{Reagents}

(+)-Catechin (C), (-)-epictechin (EC), coumaric acid (Cou A), caffeic acid (Caf A), protocatechuic acid (Prot A), gallic acid (Gal A), isoamyl acetate $(A)$, ethyl hexanoate $(H)$, damascenone $(D)$, 4-ethylguaiacol (ETG), 3-hexanol (3H) and 4-ethylphenol (ETF) were purchased from Sigma-Aldrich (Steinheim, Germany).

\subsection{Sample preparation}

The compounds were dissolved in mineral water at $\mathrm{pH} 3.6\left(\mathrm{H}_{3} \mathrm{PO}_{4}\right.$ : Analyticals Carlo Erba, Milano, Italy). In all experiments, whether the sample to be tested corresponds to an individual phenolic compound or to a mixture of phenolic compounds, the final total concentration was $2 \mathrm{~g} \mathrm{~L}^{-1}$, so the total amount of stimulus is constant in all the tasted samples. For example, gallic acid was tasted alone at a concentration of $2 \mathrm{~g} \mathrm{~L}^{-1}$, protocatechuic acid was tasted alone at a concentration of $2 \mathrm{~g} \mathrm{~L}^{-1}$, and when the mixture of both was tasted, the final concentration of the mixture was also $2 \mathrm{~g} \mathrm{~L}^{-1}\left(1 \mathrm{~g} \mathrm{~L}^{-1}\right.$ gallic acid $+1 \mathrm{~g} \mathrm{~L}^{-1}$ protocatechuic acid). The same procedure has been applied to the rest of the mixtures evaluated.

Volatile compounds were used in this study to evaluate their possible impact on the mouthfeel characteristics of the phenolic compounds. They were selected taking into account their contribution on the wine aroma and their sensory description (herb, fruit, wood, species, etc.). Their concentrations were chosen considering their olfactory detection thresholds and their usual concentration in wines (Cedrón Fernandez, 2004). The concentrations used were: damascenone $\left(5 \mu \mathrm{g} \mathrm{L}^{-1}\right)$, 3hexanol $\left(1 \mu \mathrm{g} \mathrm{L}^{-1}\right)$, 4-ethylguaiacol $\left(35 \mu \mathrm{g} \mathrm{L}^{-1}\right)$, isoamyl acetate $\left(160 \mu \mathrm{g} \mathrm{L}^{-1}\right)$, ethyl hexanoate $\left(190 \mu \mathrm{g} \mathrm{L}^{-1}\right)$, and 4-ethylphenol $\left(150 \mu \mathrm{L} \mathrm{L}^{-1}\right)$.

\subsection{Sensory analysis}

The sensory panel included up to nineteen wine-taster experts ( 8 men and 11 women) aged between 23 and 60 years old. All of them have high experience describing organoleptic sensations. The panelists attended four preliminary training sessions in order to familiarize with the sensory attributes and to standardize the use of the terms and the scale. Samples were tested in a tasting room in individual booths in the Polytechnic University of Valencia (Valencia, Spain). In the training sessions, panelists were asked to mark when they began to feel some stimuli and to rate the samples on ascending order (see below for scale details). They also had to order the samples according the type of taste or oral sensation. A duo-trio test was also performed between samples using aluminum potassium sulfate and tannic acid as astringency standards. Subjects were asked for the perceived sensations, if they were similar or different and why.

The following solutions were used for the training sessions: aqueous solutions of quinine hydrochloride dihydrate $\left(0.025-0.1 \mathrm{~g} \mathrm{~L}^{-1}\right)$ to carry out the assay of bitterness, aluminum potassium sulfate $\left(0.25-1 \mathrm{~g} \mathrm{~L}^{-1}\right)$ and tannic acid $\left(0.175-1.5 \mathrm{~g} \mathrm{~L}^{-1}\right)$ for the astringency, tartaric acid (0.125-1 $\left.\mathrm{g} \mathrm{L}^{-1}\right)$ for the sourness, sucrose $\left(1-10 \mathrm{~g} \mathrm{~L}^{-1}\right)$ for the sweetness, $\mathrm{NaCl}\left(0.25-1 \mathrm{~g} \mathrm{~L}^{-1}\right)$ for the saltiness and sodium L-glutamate 1hydrate $\left(0.25-1 \mathrm{~g} \mathrm{~L}^{-1}\right)$ for the umami taste. Moreover, in order to familiarize the subjects with other mouthfeel properties such as smoothness, mouth-fullness, body and viscosity some tasting exercises were carried out using glycerol (5-20 $\mathrm{g} \mathrm{L}^{-1}$ ) and aqueous gelatine solutions (0.25$0.5 \%$ ). Subjects were asked to mark when they began to feel some stimuli and to rate the samples on ascending scale. They also had to order the samples according the type of taste or oral sensation. A duo-trio test was also performed between samples using aluminum potassium sulfate and tannic acid as astringency standards. Subjects were asked for the perceived sensations, if they were similar or different and why.

The panelists took a $5 \mathrm{~mL}$ sample in their mouth and $15 \mathrm{~s}$ afterward they rate the intensity and quality. The persistence was evaluated $2 \mathrm{~min}$ after spitting the samples out. The panelists repeated this protocol two times per sample to assign the final value for each attribute. Panelists rinsed with deionized water and waited for 2 min between samples. They were not paid and they did not know the nature of the samples.

A Labeled Magnitude Scale (LMS) was used in the sensory analysis (Green et al., 1996). This scale combines features of ratio and category scales, allowing accurate comparisons of perceived intensities across individuals. It consists in a semantic scale of perceptual intensity 
characterized by a quasi-logarithmic spacing of the verbal labels from 0 to 100 (barely detectable $\sim 2$; weak $\sim 7$; moderate $\sim 16$; strong 34; very strong $\sim 50$; strongest imaginable $\sim 100$ ). The intensity and persistence of astringency and bitterness and also different mouthfeel characteristics were evaluated. Drying, harsh, unripe, dynamic and velvety sensations were evaluated according to the hierarchically structured vocabulary of mouthfeel sensations proposed by Gawel et al. (2000). Moreover, as a complement to the quantitative descriptive analysis, panelists were asked to include their own terminology to describe the perceived mouthfeel sensations.

\subsection{Statistical analysis}

In order to determine the consistency of the trained panel along the time, one-way analysis of variance $(\alpha=0.01)$ was performed with the scores given by the panelists for an epicatechin standard solution at five different dates along the study.

Furthermore, unsupervised pattern recognition methods are widely applied in order to observe trends in the data indicating relationships between samples and/or between variables. The unsupervised method used for data analysis was principal components analysis (PCA), which was applied to the covariance matrix of the original variables.

The SPSS 13.0 for Windows software package (SPSS, Inc., Chicago, IL) was used for data processing.

\section{Results and discussion}

In this kind of work the choice of the medium where the compounds will be dissolved is a cause for concern. Preliminary tests were performed in wine like media with 12 to $4 \%$ of ethanol. In all cases, the alcohol elicited a burning sensation that masked all other sensory perceptions and this medium was finally discarded. Obviously, in a real wine, this sensation is not so perceived due to the complexity of the wine matrix allowing the suitable equilibrium of the components. More complex media like white wines treated with activated carbon were also discarded at this time to avoid the variability that the remained compounds could exert on the different phenolics tested that could affect their astringent sub-qualities. Finally, aqueous solutions adjusted at pH 3.6 were used in the study. Different acids were tested for $\mathrm{pH}$ adjustment (hydrochloric, lactic, citric, tartaric and phosphoric acids) to know their sensation of sourness or even their possible impact on the astringency. Phosphoric acid was shown as the most neutral acid when it was dissolved in water ( $\mathrm{pH}$ 3.6) and finally it was chosen.

Also, preliminary tests were carried out to estimate the most appropriate concentration of the phenolic compounds to be tasted. We found that $2 \mathrm{~g} \mathrm{~L}^{-1}$ of these phenolic compounds showed a moderate feeling allowing an upper and lower perception. Higher concentrations induced strong saturation in the mouth. On the contrary, lesser concentration made evaluation of some of the astringency sub-qualities difficult. Furthermore, this concentration becomes appropriate since a typical young red wine may have a total content in tannins (that is a total content of potential-astringency source) of about $2 \mathrm{~g} \mathrm{~L}^{-1}$. Other authors have used concentrations of the same order to evaluate bitterness and astringency of catechins (Kallithraka et al., 1997; Robichaud \& Noble, 1990). To better characterize and to compare the sensations elicited by the different phenolic compounds, all were tested at the same concentration although their concentrations in wine could be substantially different.

Volatile compounds were used in this study to evaluate their possible impact on the mouthfeel characteristics of the phenolic compounds. They were selected taking into account their contribution on the wine aroma and their sensory description (herb, fruit, wood, species, etc.). Their concentrations were chosen considering their olfactory detection thresholds and their usual concentration in wines (Cedrón Fernandez, 2004).
A Labeled Magnitude Scale (LMS) was used for rating the intensity of the perceived sensations. This scale combines features of ratio and category scales, allowing accurate comparisons of perceived intensities across individuals (Green et al., 1996).

\subsection{Panelist reliability along the time}

As can be seen in Table 1 no significant differences $(p>0.01)$ were observed in the scores given by the panelist for the sensory parameters of an epicatechin standard solution along the time. The discrepancy between the values assigned for each date (between groups) by the sensory panel did not present significant differences demonstrating that panelists could repeat the astringency sub-qualities scores on different instances. Velvety and persistence attributes showed the lowest levels of significance indicating that these parameters could be the most difficult for evaluating along the time although no significant differences between dates were found in the present study.

\subsection{Sensory profile of phenolic acids and catechins}

The sensory profile of the studied phenolic acids and catechins is shown in Fig. 1. It can be observed that phenolic acids (coumaric, caffeic, gallic and protocatechuic acids) produce astringency and slight bitterness. These results are in good agreement with the study of Hufnagel and Hofmann (2008), which stated that hydroxybenzoic and hydroxycinnamic acids could act as sensory-active compounds contributing to wine astringency. The phenolic acids tested individually showed similar scores for the different attributes studied. Basically, at this concentration, they were more astringent than bitter with an over-moderate astringent intensity and, it could say, a weak persistence. The astringency sub-qualities velvety, drying, harsh and unripe were between 'weak' and 'moderate'. Dynamic quality was, in general, barely detectable. When the hydroxycinnamic acids are tasted together (HC), and the sensory perception elicited is compared to the hydroxybenzoic acids also tasted as a mixture (HB), it can be observed

Table 1

Results of the one-way ANOVA performed with the scores given by the panelists for an epicatechin standard solution $\left(2 \mathrm{~g} \mathrm{~L}^{-1}\right)$ along the time. Between groups: variation between different dates.

\begin{tabular}{|c|c|c|c|c|c|c|}
\hline & & Sum of squares & $\mathrm{gl}$ & Mean square & $\mathrm{F}$ & Sig. \\
\hline \multirow[t]{3}{*}{ Intensity } & Between groups & 194.592 & 4 & 48.648 & 0.654 & 0.628 \\
\hline & Within groups & 3051.778 & 41 & 74.434 & & \\
\hline & Total & 3246.37 & 45 & & & \\
\hline \multirow[t]{3}{*}{ Bitterness } & Between groups & 243.593 & 4 & 60.898 & 0.51 & 0.728 \\
\hline & Within groups & 4893.211 & 41 & 119.347 & & \\
\hline & Total & 5136.804 & 45 & & & \\
\hline \multirow[t]{3}{*}{ Velvety } & Between groups & 1193.591 & 4 & 298.398 & 1.691 & 0.171 \\
\hline & Within groups & 7233.822 & 41 & 176.435 & & \\
\hline & Total & 8427.413 & 45 & & & \\
\hline \multirow[t]{3}{*}{ Drying } & Between groups & 147.001 & 4 & 36.75 & 0.588 & 0.673 \\
\hline & Within groups & 2563.956 & 41 & 62.536 & & \\
\hline & Total & 2710.957 & 45 & & & \\
\hline \multirow[t]{3}{*}{ Harsh } & Between groups & 54.589 & 4 & 13.647 & 0.194 & 0.94 \\
\hline & Within groups & 2886.889 & 41 & 70.412 & & \\
\hline & Total & 2941.478 & 45 & & & \\
\hline \multirow[t]{3}{*}{ Unripe } & Between groups & 215.47 & 4 & 53.867 & 0.589 & 0.672 \\
\hline & Within groups & 3746.9 & 41 & 91.388 & & \\
\hline & Total & 3962.37 & 45 & & & \\
\hline \multirow[t]{3}{*}{ Dynamic } & Between groups & 22.314 & 4 & 5.579 & 0.136 & 0.968 \\
\hline & Within groups & 1676.056 & 41 & 40.879 & & \\
\hline & Total & 1698.37 & 45 & & & \\
\hline \multirow[t]{3}{*}{ Persistence } & Between groups & 207.551 & 4 & 51.888 & 1.108 & 0.366 \\
\hline & Within groups & 1919.167 & 41 & 46.809 & & \\
\hline & Total & 2126.717 & 45 & & & \\
\hline
\end{tabular}

Within groups: variation between the values assigned by each panelist for the same date Between groups: variation between different dates. 
that they show very similar values for all the attributes except for drying. Nevertheless, the mixture of both types of phenolic acids, that is to say hydroxybenzoic and hydroxycinnamic acids ( $\mathrm{HB}+\mathrm{HC}$ ) shows a noticeable increase in the perception of most of the attributes, and mainly in bitterness. This issue will be discussed in the next section.

The sensory profile of catechins is also shown in Fig. 1. As it was already reported by other authors (Kallithraka et al., 1997; Robichaud \& Noble, 1990; Thorngate \& Noble, 1995), epicatechin (EC) was more bitter and astringent than catechin (C). It is also noticeable that epicatechin showed longer persistence and higher values for the attributes drying, unripe, velvety and harsh. Although velvety is a positive sensation, in general arguably epicatechin is more 'unpleasant' than catechin. The astringent intensity of the four phenolic acids and the epicatechin was very similar but that was not the case for the catechin, which has lower values. The difference between EC and individual phenolic acid sensory profiles was mainly due to their bitterness. In relation to the compounds tasted individually, the mixture C + EC showed an increase in the perception of the attributes except in velvety.

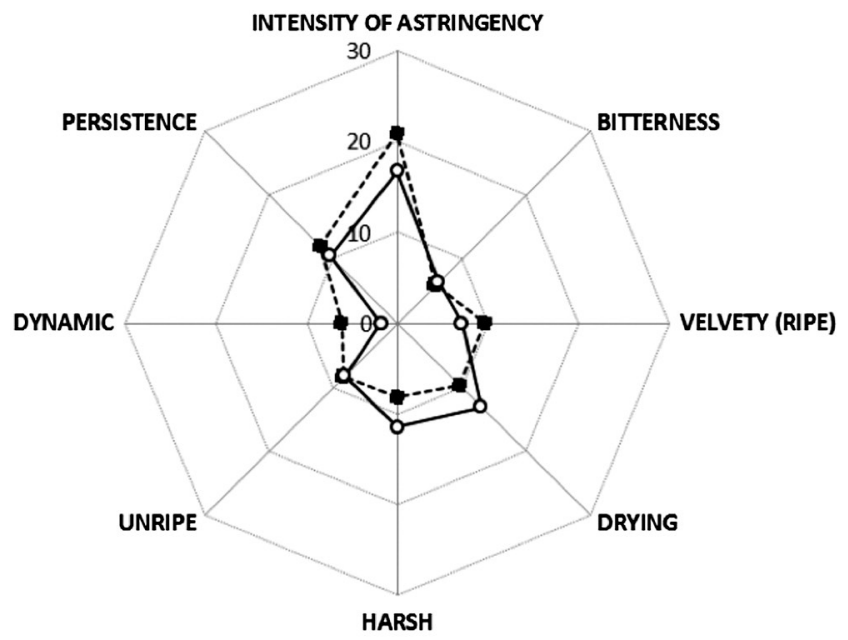

----- Coumaric acid $\quad-0-$ Caffeic acid

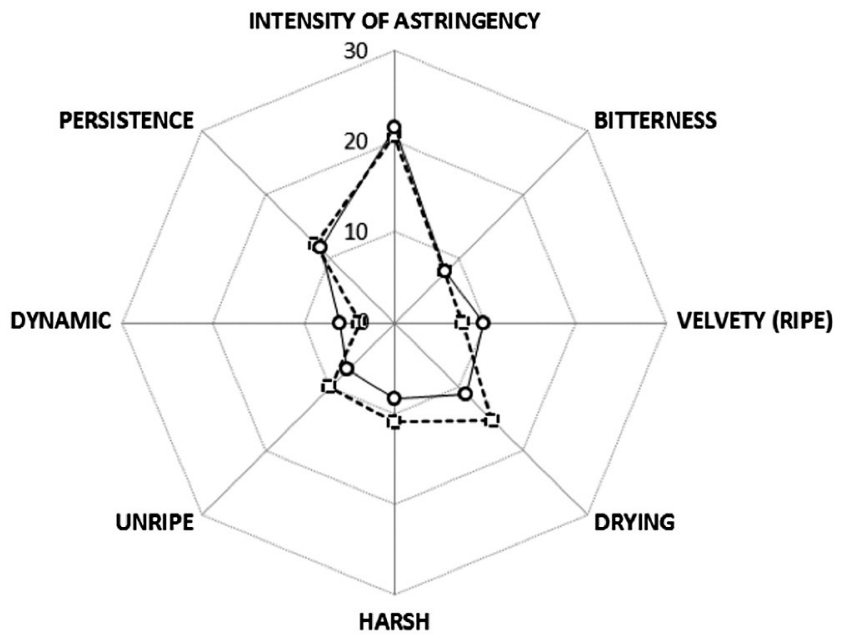

---t--- Gallic acid —o- Protocatechuic acid

\subsection{Sensory profile of mixtures: the 'co-astringency' effect}

The common definition of synergism refers to mixtures that have a stronger perceived intensity than the sums of the perceived intensities of the mixture components. That is to say:

Component $\mathrm{A}$ (at $\mathrm{n}$ concentration) has $x$ perceived intensity.

Component $\mathrm{B}$ (at $\mathrm{n}$ concentration) has $y$ perceived intensity.

The mixture $\mathrm{A}+\mathrm{B}$ (final concentration $2 \mathrm{n}$ ) has $z$ perceived intensity. If $z>x+y$, synergism is said to occur.

In this definition of synergism, the perceived astringency $z$ is compared to a theoretical predicted intensity (the sum of $x+y$ ), assuming that perceived intensity of the substances is a linear function of its concentration although it is not necessarily like that. To avoid comparisons with "predicted" perceived intensities, in our study the mixtures and the unmixed components were tasted as the same concentration. Furthermore, the use of scales with ratio properties (that is the case for the Labeled Magnitude Scale used in this study) has proven to be successful for demonstrating synergism (Rifkin \& Bartoshuk, 1980).
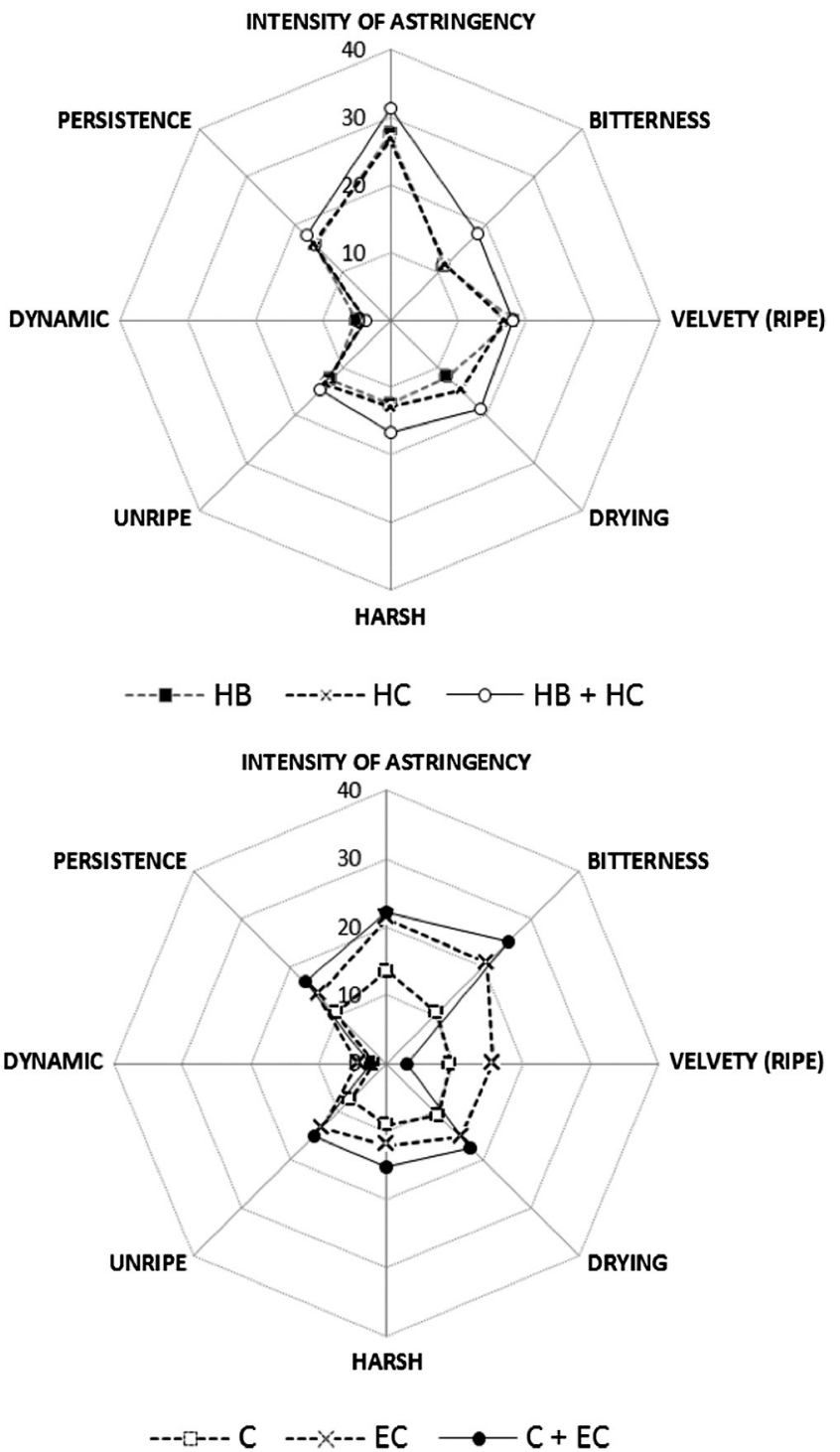

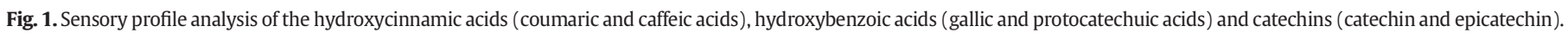
HB: hydroxybenzoic acids; HC: hydroxycinnamic acids; C: catechin; EC: epicatechin. 
In our study on mixtures of phenolic compounds, all mixtures were tasted at the same concentration as the individual compounds, so the total amount of stimulus is constant in all the tasted samples. For example, gallic acid was tasted alone at a concentration of $2 \mathrm{~g} \mathrm{~L}^{-1}$, protocatechuic acid was tasted alone at a concentration of $2 \mathrm{~g} \mathrm{~L}^{-1}$, and when the mixture of both was tasted, the final concentration of the mixture was also $2 \mathrm{~g} \mathrm{~L}^{-1}\left(1 \mathrm{~g} \mathrm{~L}^{-1}\right.$ gallic acid $+1 \mathrm{~g} \mathrm{~L}^{-1}$ protocatechuic acid). The same procedure has been applied to the rest of the mixtures evaluated. Fig. 2 shows the psychophysical functions for astringency and bitterness when phenolic substances were tested alone and when they were tested as mixtures. Mixtures tasted correspond to hydroxybenzoic acids (mixture of gallic and protocatechuic acids), hydroxycinnamic acids (mixture of coumaric and caffeic acids), phenolic acids (mixture of gallic, protocatechuic, coumaric and caffeic acids) and catechins (mixture of catechin and epicatechin). As shown in Fig. 2, the perceived intensities of bitterness and astringency of the mixtures (final concentration $2 \mathrm{~g} \mathrm{~L}^{-1}$ ) are higher than the perceived intensities of the components tasted individually at the same concentration (i.e. $2 \mathrm{~g} \mathrm{~L}^{-1}$ ), indicating the existence of synergism. If synergism would not occur, the perceived intensities of the mixtures should be equal or lower than the perceived intensity of, at least, one of the components tasted individually at the same concentration.

This pattern was observed for hydroxybenzoic acids and hydroxycinnamic acids, for the mixture of both hydroxybenzoics and hydroxycinnamics and also for catechins. Hence, mixtures of phenolic acids and mixtures of catechins showed synergism for astringency and bitterness since the perceived intensities of the mixtures exceeded the perceived intensities of the components evaluated at the same concentration.

Furthermore, the synergistic effect could also be figured out in the principal component analysis (PCA).
The PCA was applied as an unsupervised pattern recognition method to observe trends in the data and to indicate relationships between phenolic substances and/or between the sensory attributes. Fig. 3 shows the projection of the samples on the plane defined by the first and second principal components (Fig. 3a) and also the corresponding loading plot (Fig. 3b). The first and second principal components describe $89.4 \%$ of the variability ( $59.8 \%$ and $29.6 \%$, respectively). Unripe, harsh, persistence and drying sensations presented the most similar pattern (Fig. 3b). The most negative values in PC1 correspond to the original variables "Bitterness" and "Intensity of astringency". In Fig. 3a, individual phenolic acids (coumaric acid: Cou A, caffeic acid: Caf A, gallic acid: Gal A and protocatechuic acid: Prot A) and catechin present a homogeneous pattern, which correspond to the lowest values of almost all sensory attributes. Epicatechin (EC) presented a different pattern in the PC1 clearly distinguishable from the other samples tasted individually.

A general tendency of the mixtures to be placed towards the left zone of the PCA plot can be observed. The mixture consisting of hydroxycinnamic and hydroxybenzoic acids $(\mathrm{HB}+\mathrm{HC})$ showed more negative values in PC1 than mixtures containing hydroxycinnamic acids (HC) and hydroxybenzoic acids (HB) and, in turn, these mixtures showed more negative values than their respective components (i.e. Cou A, Caf A, Gal A and Prot A) tasted individually at the same concentration as the total concentration of the mixtures (i.e. $2 \mathrm{~g} \mathrm{~L}^{-1}$ ). The scores corresponding to the mixtures tend to be placed closer to the variables "Intensity of astringency" and "Velvety" as the complexity of the mixture increases. Thus, the PCA has allowed us to easily visualize the synergistic effect discussed above.

When catechin (C) and epicatechin (EC) were tasted together $(\mathrm{C}+\mathrm{EC})$, the perception of the mouthfeel characteristics of the mixture solution was quite different to that elicited for the solutions containing
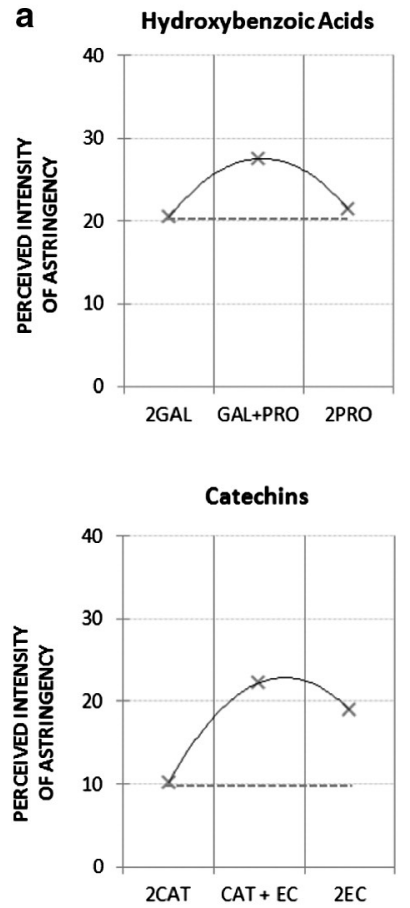

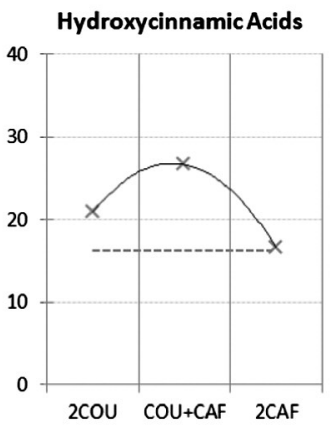

Hydroxybenzoics + Hydroxycinnamics

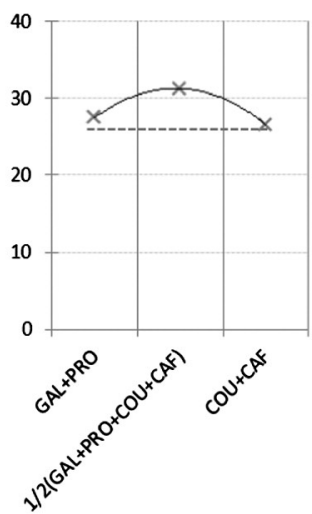

b
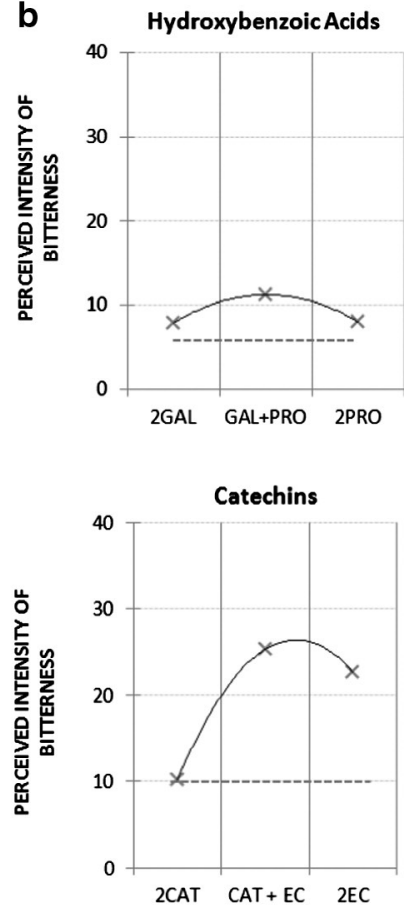

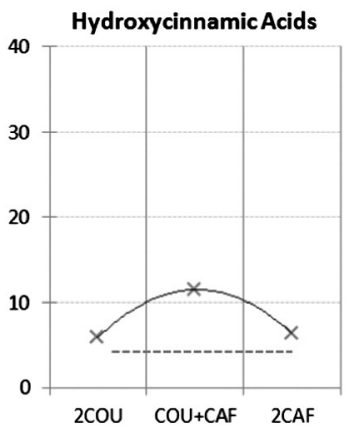

Hydroxybenzoics + Hydroxycinnamics

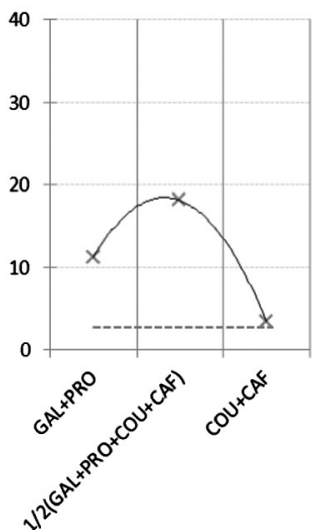

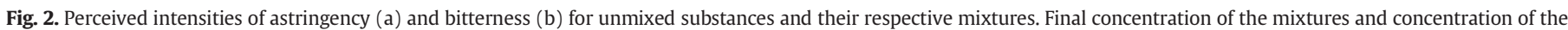
components tasted alone were $2 \mathrm{~g} \mathrm{~L}^{-1}$. Epicatechin (EC), catechin (CAT), coumaric acid (COU), protocatechuic acid (PRO), gallic acid (GAL), caffeic acid (CAF). 

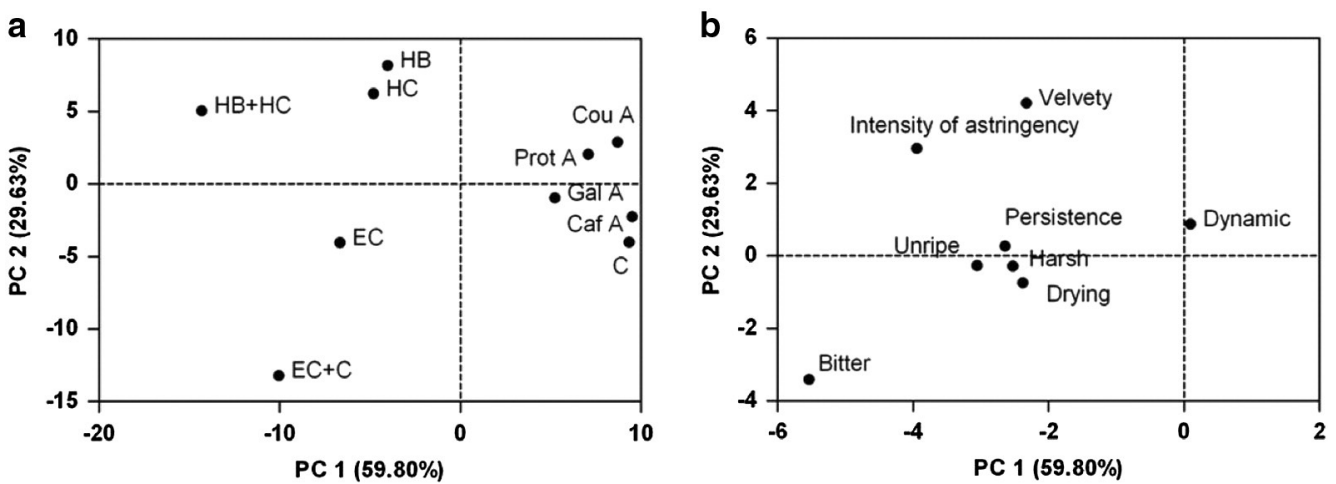

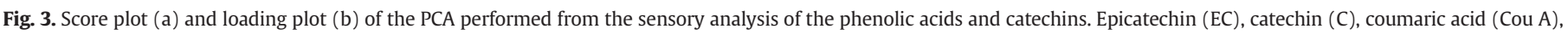

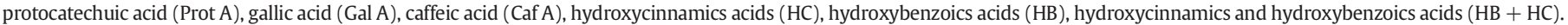

the components alone. A shift towards negative zones of the PCA can be observed and the scores approach to the original variable bitterness.

The hydroxycinnamics and hydroxybenzoics acids ( $\mathrm{HB}+\mathrm{HC})$ were more related to "Intensity of astringency" and also "Velvety" variables than catechin mixture $(\mathrm{EC}+\mathrm{C})$, which was more related to "Bitter" sensation.

The synergistic phenomenon insists on the importance of the phenolic compound structure for determining the astringency and bitterness qualities. Classically, astringency is linked to the capacity of phenolic compounds to bind proteins, which is related to the ability of these compounds to act as multidentate ligands that bind simultaneously to different points of the protein. Thereby a possibly higher variability of multidentate ligands due to the differences on the phenolic structures could facilitate the protein binding. It should be also considered that the affinity of these compounds to bind to different families of salivary proteins and the possibility to activate receptors could be involved in the development of different mouthfeel characteristics (Gibbins \& Carpenter, 2013; Soares et al., 2011). As a result, when the matrix contains different astringent substances this may develop a synergism in the perceived intensities. To our knowledge, this is the first time that astringency synergism between phenolic compounds has been described.

We have adopted the term 'co-astringency' to name the synergism on the astringent perception when the perceived intensity of the mixture of two compounds is higher than the perceived intensities of the components when they are tasted individually at the same concentration (total amount of stimulus is constant in all the tasted samples). Thus, we make a difference with the common definition of synergism, which compares the perceived intensity of a mixture with a theoretical expected value of intensity obtained from the sum of the perceived intensities of each of the component tasted individually at half of the total concentration of the mixture solution.

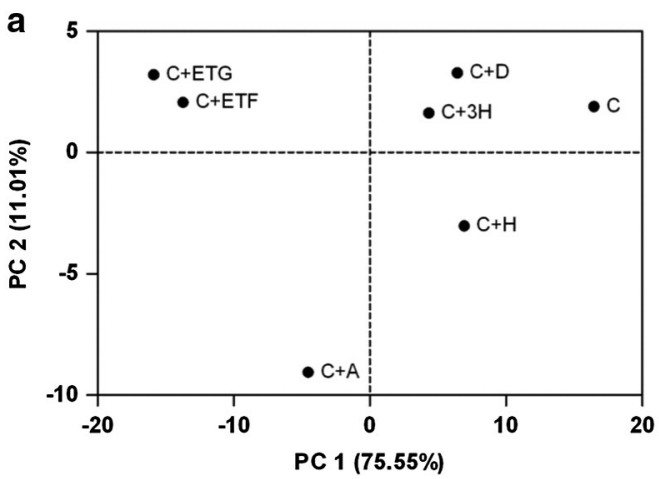

\subsection{Influence of volatile compounds in the astringent perception of catechins}

It has been stated that the addition of volatile fruity extracts could affect the astringency and the bitterness perception of wine (SaenzNavajas et al., 2010). Catechin and epicatechin were tasted individually and in the presence of pleasant and unpleasant aromas: damascenone (D), ethyl hexanoate $(\mathrm{H})$, and isoamyl acetate $(\mathrm{A})$ as pleasant aromas and 3-hexanol (3H), 4-ethylphenol (ETF), and 4-ethylguaiacol (ETG) as unpleasant aromas. In general, these types of aromas are very significant in wines, the three first-mentioned are fruity aromas (strawberry, pineapple, banana, etc....) and the latest ones are considered as unpleasant aromas (smoked, leather, etc....).

Figs. 4 and 5 show the projections of the samples on the planes defined by the first and second principal components (Figs. 4a and 5a) and also the corresponding loading plots (Figs. $4 \mathrm{~b}$ and $5 \mathrm{~b}$ ) for catechin and for epicatechin respectively. In both figures, the variability explained in PC1 was ca.75\%. Regarding catechin (Fig. 4), when it was tasted in the presence of the volatile compounds, the scores tend to the more negative values of the PC1, where the original variables (except velvety) are placed. The sensory parameter most affected by the addition of volatile compounds was persistence (highest absolute value in the first principal component loading). This attribute had a high contribution to PC1, allowing us to distinguish between the catechin solutions when they were tasted in the presence or in the absence of aromas. The increment was especially important in the case of C + ETG, C + ETF and C + A. This pattern suggests that this parameter may have a relationship with these aromas.

Regarding epicatechin (Fig. 5), samples tasted with aromas tend to the right zone of the PC1, towards higher values of persistence and intensity of astringency, whether they were good and bad aromas. The increase of the persistence was more intense when pleasant aromas were used in the flavanol-aroma mixture (EC + H, EC + A and EC + D).

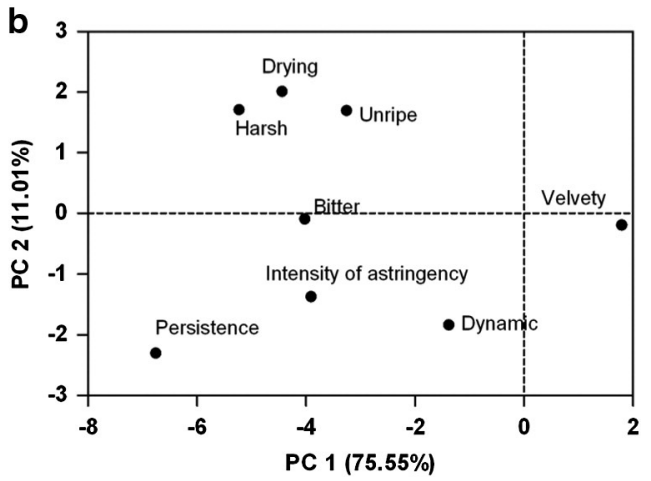

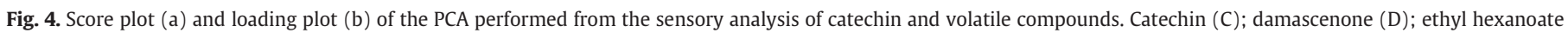
$(\mathrm{H})$; isoamyl acetate $(\mathrm{A})$; 3-hexanol (3H); 4-ethylphenol (ETF) and 4-ethylguaiacol (ETG). 

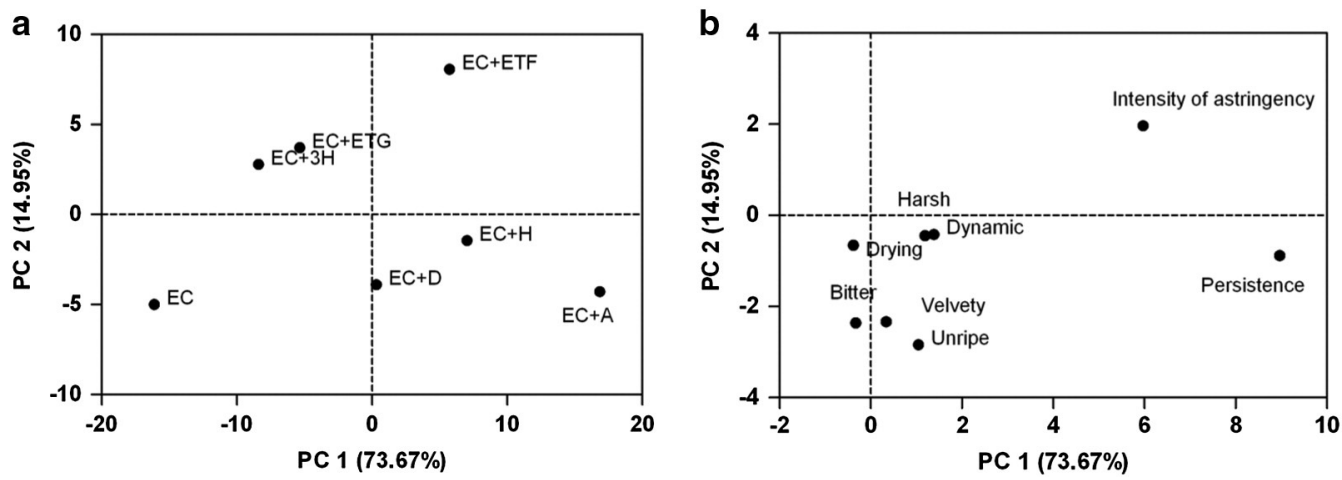

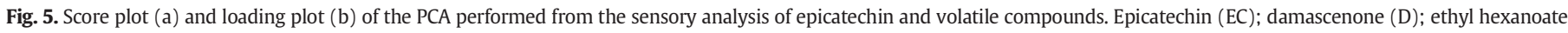
(H); isoamyl acetate (A); 3-hexanol (3H); 4-ethylphenol (ETF) and 4-ethylguaiacol (ETG).

The increment in the intensity of the astringency was especially important in the case of epicatechin tasted in the presence of unpleasant aromas (mainly with ETF). A clear separation was observed regarding the effect of the type of aroma (i.e. pleasant or unpleasant) in PC2.

Thus, it can be said that the volatile compounds increased the intensity of the astringent perception of the flavanol solutions, and also its persistence.

\section{Conclusions}

The results obtained allowed us to characterize and compare the astringent sub-qualities of epicatechin, catechin, coumaric acid, caffeic acid, protocatechuic acid and gallic acid. The phenolic acids tested individually were basically more astringent than bitter with an overmoderate astringent intensity. As for the sensory profile of catechins, EC was more bitter and astringent than $\mathrm{C}$, and it showed longer persistence. In general, EC was more 'unpleasant' than C. The astringent intensity of the four phenolic acids and the EC was very similar but that was not the case for $\mathrm{C}$, which had lower values. The difference between individual phenolic acids and EC was mainly due to its bitterness.

A synergistic astringent effect was observed and described for the first time for these compounds. It has been named 'co-astringency' effect, consisting of the increase observed on the astringency when different tannic substances are tasted together versus these tannic substances tasted individually, at the same final concentration. This term has been coined for distinguishing it to the common definition of synergism, which compares the perceived intensity of a mixture with a theoretical expected value of intensity obtained from the sum of the perceived intensities of the components tasted individually at half of the total concentration of the mixture solution.

Moreover, the relation between aromas and astringency has been underlined. The astringent perception may be modulated by volatile compounds. In general, the addition of volatile compounds to the phenolic solutions showed an increase on the rate intensities of the astringent sub-qualities.

As result of the synergism observed, this work opens an interesting future research area in understanding the perception of the astringency. However, comprehensive studies should be made in order to evaluate other factors, such as different concentrations and combinations of phenolic compounds.

\section{Acknowledgments}

Thanks are due to the Spanish MICINN (Project ref. AGL2011-30254C02) for the financial support. The authors also thank the panelists, particularly Dra. Álvarez-Cano, for their contribution. R. Ferrer-Gallego thanks Fundación Alfonso Martín Escudero for the postdoctoral fellowship. J.M. Hernández-Hierro thanks the Spanish MICINN for the Juan de la Cierva contract (JCI-2011-09201).

\section{References}

Cala, O., Dufourc, E. J., Fouquet, E., Manigand, C., Laguerre, M., \& Pianet, I. (2012). The colloidal state of tannins impacts the nature of their interaction with proteins: The case of salivary proline-rich protein/procyanidins binding. Langmuir, 28(50), 17410-17418.

Cedrón Fernandez, M. T. (2004). Estudio analítico de compuestos volátiles en vino. Caracterización quimiométrica de distintas denominaciones de origen. La Rioja: Universidad de La Rioja.

Challacombe, C. A., Abdel-Aal, E. S. M., Seetharaman, K., \& Duizer, L. M. (2012). Influence of phenolic acid content on sensory perception of bread and crackers made from red or white wheat. Journal of Cereal Science, 56(2), 181-188.

Charles, M., Poinot, P., Texier, F., Arvisenet, G., Vigneau, E., Mehinagic, E., et al. (2013). The 'Mouth to Nose Merging System': A novel approach to study the impact of odour on other sensory perceptions. Food Quality and Preference, 28(1), 264-270.

Cliff, M. A. Stanich, K., Edwards, J. E. \& Saucier, C. T. (2012). Adding grape seed extract to wine affects astringency and other sensory attributes. Journal of Food Quality, 35(4), 263-271.

de Freitas, V., \& Mateus, N. (2012). Protein/polyphenol interactions: Past and present contributions. Mechanisms of astringency perception. Current Organic Chemistry, 16(6), 724-746.

Dinnella, C., Recchia, A., Vincenzi, S., Tuorila, H., \& Monteleone, E. (2010). Temporary modification of salivary protein profile and individual responses to repeated phenolic astringent stimuli. [article]. Chemical Senses, 35(1), 75-85.

Dufour, C., \& Bayonove, C. L. (1999). Interactions between wine polyphenols and aroma substances. An insight at the molecular level. [article]. Journal of Agricultural and Food Chemistry, 47(2), 678-684.

Ferrer-Gallego, R., García-Marino, M., Hernández-Hierro, J. M., Rivas-Gonzalo, J. C., \& Escribano-Bailón, M. T. (2010). Statistical correlation between flavanolic composition, colour and sensorial parameters in grape seed during ripening. Analytica Chimica Acta, 660(1-2), 22-28.

Ferrer-Gallego, R., Gonçalves, R., Rivas-Gonzalo, J. C., Escribano-Bailon, M. T., \& de Freitas, V. (2012). Interaction of phenolic compounds with bovine serum albumin (BSA) and alpha-amylase and their relationship to astringency perception. Food Chemistry, 135(2), 651-658.

Ferrer-Gallego, R., Hernandez-Hierro, J. M., Rivas-Gonzalo, J. C., \& Escribano-Bailon, M. T. (2011). Multivariate analysis of sensory data of Vitis vinifera L. cv. Graciano during ripening. Correlation with the phenolic composition of the grape skins. Cyta-Journal of Food, 9(4), 290-294.

Gawel, R., Oberholster, A., \& Francis, I. L. (2000). A 'Mouth-feel Wheel': Terminology for communicating the mouth feel characteristics of red wine. Australian Journal of Grape and Wine Research, 6(3), 203-207.

Gibbins, H. L., \& Carpenter, G. H. (2013). Alternative mechanisms of astringency - What is the role of saliva? Journal of Texture Studies, 44(5), 364-375.

Goldner, M. C., Lira, P. D., van Baren, C., \& Bandoni, A. (2011). Influence of polyphenol levels on the perception of aroma in Vitis vinifera cv. Malbec wine. South African Journal of Enology and Viticulture, 32(1), 21-27.

Green, B. G., Dalton, P., Cowart, B., Shaffer, G., Rankin, K., \& Higgins, J. (1996). Evaluating the 'labeled magnitude scale' for measuring sensations of taste and smell. Chemical Senses, 21(3), 323-334.

Hufnagel, J. C., \& Hofmann, T. (2008). Orosensory-directed identification of astringent mouthfeel and bitter-tasting compounds in red wine. Journal of Agricultural and Food Chemistry, 56(4), 1376-1386.

Jones, P. R., Gawel, R., Francis, I. L., \& Waters, E. J. (2008). The influence of interactions between major white wine components on the aroma, flavour and texture of model white wine. Food Quality and Preference, 19(6), 596-607.

Jung, D. M., de Ropp, J. S., \& Ebeler, S. E. (2000). Study of interactions between food phenolics and aromatic flavors using one- and two-dimensional H-1 NMR spectroscopy. Journal of Agricultural and Food Chemistry, 48(2), 407-412.

Kallithraka, S., Bakker, J., \& Clifford, M. N. (1997). Evaluation of bitterness and astringency of $(+)$-catechin and (-)-epicatechin in red wine and in model solution. Journal of Sensory Studies, 12(1), 25-37.

Kora, E. P., Latrille, E., Souchon, I., \& Martin, N. (2003). Texture-flavor interactions in low fat stirred yogurt: How mechanical treatment, thickener concentration and aroma concentration affect perceived texture and flavor. Journal of Sensory Studies, 18(5) 367-390. 
Lawrence, G., Salles, C., Septier, C., Busch, J., \& Thomas-Danguin, T. (2009). Odour-taste interactions: A way to enhance saltiness in low-salt content solutions. Food Quality and Preference, 20(3), 241-248.

Morita, K., Narukawa, M., \& Hayashi, Y. (2007). Evaluation of taste intensity and quality of umami substances and the synergistic effect. Chemical Senses, 32(2), J1-J27.

Peleg, H., Gacon, K., Schlich, P., \& Noble, A. C. (1999). Bitterness and astringency of flavan3-ol monomers, dimers and trimers. Journal of the Science of Food and Agriculture, 79(8), 1123-1128.

Prescott, J., Ripandelli, N., \& Wakeling, I. (2001). Binary taste mixture interactions in PROP non-tasters, medium-tasters and super-tasters. Chemical Senses, 26(8), 993-1003.

Rifkin, B., \& Bartoshuk, L. M. (1980). Taste synergism between monosodium glutamate and disodium 5'-guanylate. Physiology Behavior, 24(6), 1169-1172.

Robichaud, J. L., \& Noble, A. C. (1990). Astringency and bitterness of selected phenolics in wine. Journal of the Science of Food and Agriculture, 53(3), 343-353.
Saenz-Navajas, M. -P., Campo, E., Fernandez-Zurbano, P., Valentin, D., \& Ferreira, V. (2010). An assessment of the effects of wine volatiles on the perception of taste and astringency in wine. Food Chemistry, 121(4), 1139-1149.

Schwarz, B., \& Hofmann, T. (2008). Is there a direct relationship between oral astringency and human salivary protein binding? European Food Research and Technology, 227(6), 1693-1698.

Soares, S., Vitorino, R., Osorio, H., Fernandes, A., Venancio, A., Mateus, N., et al. (2011). Reactivity of human salivary proteins families toward food polyphenols. Journal of Agricultural and Food Chemistry, 59(10), 5535-5547.

Thorngate, J. H., \& Noble, A. C. (1995). Sensory evaluation of bitterness and astringency of 3R(-)-epicatechin and 3S(+)-catechin. Journal of the Science of Food and Agriculture, 67(4), 531-535. 